\title{
Liderazgo directivo y clima institucional: radiografía de instituciones educativas privadas del sur oriental del Perú
}

\section{Executive leadership and institutional climate: X-ray of private}

educational institutions in southern Peru

\section{Liderança executiva e clima institucional: radiografia de instituições educacionais privadas no sudeste do Peru}

\author{
Dino Barrientos Velito \\ Rodolfo Alania Pacovilca \\ dinobv@hotmail.com \\ rodolfoalania@upeu.edu.pe \\ iD https://orcid.org/0000-0001-5500-7363 \\ Universidad Adventista de Chile \\ https://orcid.org/0000-0001-9522-2471 \\ Universidad Peruana Unión
}

Recibido: 03 de enero de 2021

Aceptado: 06 de junio de 2021

\section{Resumen}

En esta investigación se ha estudiado el liderazgo directivo y el clima institucional, con el objetivo de determinar la relación de estas variables, con la participación de 172 profesores, siguiendo el diseño trasversal y los tipos: descriptivo, correlacional y transversal. Los resultados fueron generados mediante dos instrumentos: uno para cada variable, validos según los proceso y protocolos estandarizados. El análisis estadístico de correlación de Rho Spearman dejó el resultado de 0,573 y un p valor igual a $0.000(<$ $0,05)$. En conclusión, el liderazgo del director se relaciona, en forma significativa, con el clima institucional, cuya relación es directa; vale decir que, a mejor liderazgo del director, mejor o mayor será el clima institucional.

Palabras clave: clima institucional, liderazgo del director, liderazgo educativo, liderazgo situacional. 


\begin{abstract}
In this research, managerial leadership and the institutional climate have been studied, in order to determine the relationship of these variables, with the participation of 172 teachers, following the transversal design and the types: descriptive, correlational and transversal. The results were generated using two instruments: one for each variable, valid according to the standardized processes and protocols. The Rho Spearman statistical analysis of correlation left the result of 0.573 and a p value equal to $0.000(<0.05)$. In conclusion, the director's leadership is significantly related to the institutional climate, which is directly related; In other words, the better the director's leadership, the better or the greater the institutional climate.
\end{abstract}

Keywords: institutional climate, director's leadership, educational leadership, situational leadership.

\title{
Resumo
}

Nesta pesquisa, a liderança gerencial e o clima institucional foram estudados, a fim de determinar a relação dessas variáveis, com a participação de 172 professores, seguindo o desenho transversal e os tipos: descritivo, correlacional e transversal. Os resultados foram gerados por meio de dois instrumentos: um para cada variável, válido de acordo com processos e protocolos padronizados. A análise estatística de correlação de Rho Spearman deixou o resultado de 0,573 e um valor de p igual a 0,000 $(<0,05)$. Em conclusão, a liderança do diretor está significativamente relacionada ao clima institucional, que está diretamente relacionado; ou seja, quanto melhor a liderança do diretor, melhor ou maior será o clima institucional.

Palavras-chave: clima institucional, liderança do diretor, liderança educacional, liderança situacional.

\section{Introducción}

La EBR en el Perú no tiene la sintonía plena sobre el significado del liderazgo directivo (Rossi y Rossi, 2021; Córdova et al., 2021). Existe un universo donde, en forma constante, dialogan la comunicación, el liderazgo, el clima institucional, las expresiones, los gestos, el idioma, especialmente en las instituciones educativas, cuyos líderes y liderados establecen vínculos permanentes, permitiendo que las contiendas y los 
conflictos queden exentos, buscando sobre todo la paz, el entendimiento, la armonía y la esperanza para todos. Este es el ideal buscado todos los días; sin embargo, la experiencia y la realidad anuncian lo contrario. En este contexto, el liderazgo y el clima institucional hacen una alianza institucional para el bien de las instituciones y de las naciones.

El liderazgo directivo y el clima institucional constituyen dos variables muy importantes, expuestas al presente estudio, porque son vitales para la buena convivencia de los sujetos de las instituciones educativas, inclusive para la mejora del proceso enseñanza aprendizaje.

Por ejemplo, en México, ¿cómo está el clima escolar? Valdéz, Tánori, Sotelo y Ochoa (2018) realizan un estudio, en el cual tratan cuatro variables: prácticas docentes, clima social, seguridad escolar y violencia. Hacen conocer que la Reforma Integral de la Educación Básica (RIEB) no ha logrado sus objetivos diseñados; en este panorama, se informa que el $25,0 \%$ de los estudiantes aparecen en la condición de víctimas y/o agresores; el 47,2\% de los estudiantes está presente durante las agresiones. Además, se observa el acoso, la ansiedad, la depresión, las malas relaciones interpersonales, las conductas auto destructivas.

Muchos entornos escolares no son positivos, no son favorables. En los espacios escolares y laborales, la autoridad es vertical, dejando limitaciones para la expresión de "las competencias sociales e inteligencia emocional del docente", constituyéndose de esta manera en problemas gravitantes (Cortez, 2018).

Quezada (2018) estudia el liderazgo estratégico del director y el programa de tecnología educativa, en la Universidad Nacional de Trujillo, con el objetivo general: "determinar el grado de influencia que existe entre el liderazgo estratégico del director y el éxito institucional del Programa de Tecnología Educativa" (p. v). El estudio corresponde a una metodología cuantitativa, básica, de diseño correlacional y explicativa, en una población muestral de 6 profesores, determinada en forma no probabilística; los datos fueron recabados mediante un cuestionario, cuyo análisis se realizó mediante la estadística descriptiva e inferencial. Los resultados presentan el coeficiente de correlación de Pearson de $\mathrm{R}=0.941$, lo cual es altamente significativo; se concluye que el "liderazgo estratégico del director influye significativamente en el éxito institucional del Programa de Tecnología Educativa". 
En este contexto y dada la importancia sustantiva del liderazgo directivo y el clima institucional, se considera muy pertinente el estudio, cuyo objetivo es analizar la relación de las dos variables en cuestión; para cuyo efecto y mejor precisión conceptual, abordaje y significativo análisis, se consideró pertinente abordar las bases teóricas siguientes.

\section{Liderazgo directivo}

Concepciones diversas sobre el liderazgo directivo

Mejía (2015) sustenta que el liderazgo del director o liderazgo directivo deja la consigna semántica de capacidad de orientar, guiar, alinear, generación y otorgamiento de confianza, unidad, identidad, identificación, continuación. Según la concepción de Calderón (2017), el liderazgo "no es una tarea solitaria", queda definido por la función de los liderados, los demás integrantes de la institución, llegando a un liderazgo colectivo; es una competencia esencial de las personas; permite y facilita conocimiento, reflexión, diálogo, concertación, negociación. Para Quezada (2018) es la capacidad de comunicación; el factor de capacitación y gestión (Oré y Riversos, 2017).

\section{Importancia del liderazgo directivo}

El liderazgo deja muchas ventajas, muchas incidencias; por ejemplo, facilita el desarrollo de los docentes, quienes, por efecto, expresan satisfacción, compromiso, emociones positivas, autoconfianza, motivación. Las investigaciones realizadas han revelado que la dirección de la escuela (dirección escolar) siempre ha ejercido beneficios para los aprendizajes de los alumnos, resaltando uno de los factores: el compromiso de los docentes. La influencia de los directores es poderosa, aunque no sea directa sobre los aprendizajes; sin embargo, es más perceptible y destacada sobre el desarrollo de los profesores (Horn y Murillo, 2016).

El clima organizacional es favorecido por el liderazgo del director. Las instituciones educativas mejoran su calidad, gracias al liderazgo del director, si este es aceptado por los integrantes de la comunidad; además de este liderazgo depende el "planeamiento, ejecución, control y evaluación de políticas, estrategias y actividades para el cumplimiento de determinados objetivos y metas institucionales, es por ello que la pieza clave del éxito es el liderazgo directivo, mejor aún si los docentes son igualmente líderes en la actividad educativa que llevan a cabo" (Oré y Riversos, 2017, p. 9).

Según los estudios de Quezada (2018), el liderazgo del director, particularmente del liderazgo estratégico revela los beneficios: fomenta el inicio del proceso de atención a las 
necesidades, busca y estableces las modalidades de trabajo, unifica los criterios, planifica los avances, reduce la incertidumbre y une los esfuerzos, genera identidad, categoriza la información, fomenta la estrategia del liderazgo.

El liderazgo se ha constituido en un factor muy importante para las instituciones educativas, en las cuales son permisibles la innovación, la motivación, la toma de decisiones, la evaluación, la relación laboral, la cooperación, la participación plena, bajo la perspectiva de que los recursos humanos son la base de la organización (Valdivia, 2018).

Por otro lado, el liderazgo de director mejora el aprendizaje (Naira, 2018). Fomenta el diálogo y los consensos; también mejora el desempeño docente, la gestión administrativa, genera en los docentes el compromiso organizacional (Salvador \& Sánchez, 2018). Por otro lado, el liderazgo pedagógico del director mejora "práctica pedagógica de su equipo de docentes, así como el acompañamiento sistemático a los procesos pedagógicos con la finalidad de lograr las metas de aprendizaje establecidas"; también apoya, evalúa y desarrolla la calidad del docente (Chicoma, 2018). Busca la calidad educativa, "uno de los factores que hacen posible la denominada educación de calidad, es la presencia de un liderazgo directivo efectivo, asertivo, democrático, participativo, que involucre al personal docente en los planes de trabajo de modo activo y protagónico" (Maya et al., 2019, p. 115). Produce el cambio el cambio educativo (Aparicio et al., 2020); mejora el clima organizacional y la satisfacción laboral (Simbron y Sanabria, 2020), también el desempeño docente (Ordoñez et al., 2020; Gutierrez, 2020); prepara para la justicia social (Rodríguez et al., 2020).

\section{Enfoques de liderazgo directivo}

Existen varios enfoques: enfoque socio formativo para Herrera y Tobón (2017), para quienes el perfil del director escolar se construye sobre la base de las competencias directivas; Para Delhom et al. (2019), según el enfoque de los rasgos, las personas tienen diversas y sus propias características, cuyas características psicológicas y físicas; enfoque conductual es condicionado, causal e innato; para el enfoque situacional, los resultados y las conductas dependen de las situaciones y de los controles.

\section{Teorías del liderazgo directivo}

La Teoría de los rasgos aborda las características del líder, expresadas en sus dimensiones sociales, físicas, intelectuales (Robinson, 2011, en Mejía, 2015). Las 
Teorías del comportamiento han permitido la búsqueda de la capacitación a las personas (Mejía, 2015). La Teoría sobre el liderazgo de Likert, según Likert (Navarro, 2018), determina cuatro sistemas: autoritario-coercitivo (fija decisiones y directivas sin consultas), autoritario-benevolente (ejerce poca confianza), participativo (tiene confianza en sus colaboradores) y consultivo (expresa plena confianza en sus colaboradores).

\section{Clima institucional}

\section{Concepciones diversas sobre el clima institucional}

Vega (2017), en sus estudios, recoge varias concepciones; por ejemplo, el sentido de percepción de las personas sobre la institución educativa y su estructura: formal e informal, la cultura organizacional, las relaciones sociales; percepción de la comunidad educativa; calidad de relaciones interpersonales; valores, comunicación, normas, prácticas, éticas, comportamientos; pertenencia, claridad, justicia; seguridad, relaciones, enseñanza, aprendizaje, entorno.

Con el sentido de sistema complejo, es "una construcción compleja". Con el sentido de fenómeno, el clima escolar denominado fenómeno influye, incide sustancialmente (Vega, 2017). Con el sentido de factor, para González (2017) es un "conjunto de factores"; factor muy relevante; catalizador generado para mejorar el clima escolar; genera "el apoyo, la confianza y el respeto mutuo", "relaciones e interacciones sociales positivas”, la vinculación y la reducción de “conductas violentas” (Vega, 2017).

\section{Importancia del clima institucional}

El clima escolar favorece las condiciones de trabajo, también motivación (Ruiz de Durand, 2016). Para Puscán (2017) permite "el aprendizaje de reconocimiento y manejo de las emociones". El clima adecuado produce ambiente saludable, favorece las relaciones, la convivencia (Oré y Riversos, 2017); ofrece beneficios, efectos muy positivos (Cortez, 2018).

\section{Estrategias para mejorar el clima institucional}

Se requiere la concurrencia de la parte directivas, cuyos responsables realicen en forma permanente el "monitoreo, el acompañamiento y la evaluación de la práctica pedagógica". comparte su plan de acción relacionado con sus componentes: objetivos, estrategias, metas, actividades, responsables, recursos y cronograma (Còrdova, 2015); se mejora con el plan de marketing interno (Arca, 2020). 
El clima institucional es muy importante para las organizaciones y las personas quienes las componen. Siempre ha buscado diversos medios y estrategias para fortalecerlo. Ha mejorado y mejora mediante el liderazgo de servicio (Lescano, 2017); el buen clima, el positivo, tiene conexión directa con el respeto a las normas y los ideales normativos también superan las patologías sociales (Cuenca y Cáceda, 2017).

\section{Teorías y enfoques del clima institucional}

\section{Teoría de Mc Gregor}

El autor de esta teoría propuso dos teorías: Teoría X y teoría Y. Para la primera teoría, la persona es perezosa, rehúye, egocéntrica, dependiente, controlada, dogmática, dirigida, resiste al cambio; cuya administración y liderazgo son autocráticos, lineales, clásicos. En cambio, la segunda teoría supone la presencia de una persona dinámica, sensible, cooperadora, con capacidad para dirigir, con excelente iniciativa, dedicado, esforzado. Esta teoría genera una propuesta de liderazgo, encaminada a desarrollar la motivación de los docentes (Vargas y Vásquez, 2018; Madero y Rodríguez, 2018; Marlene \& Rujel, 2020).

Teoría de Sistemas. Significa que las conductas son consecuencia de otras conductas. Las conductas de los administradores generan conductas en los subordinados, dependiendo de los informes, las percepciones, las esperanzas, las capacidades, los valores. La conducta se desarrolla en la relación acción-reacción (Oré y Riversos, 2017). El comportamiento de las personas responde a ciertas reacciones administrativas. Enfoque estructural y humanista. Los aspectos objetivos (tamaño, centralización, descentralización, toma de decisiones, tecnología, autoridad, medio ambiente, interacción, características personales) determinan el clima institucional (Medina, 2018; Peralta, 2016).

\section{Metodología}

\section{Tipo y diseño del estudio}

Para los efectos de los análisis estadísticos, se han diseñado cinco modelos estadísticos, con el propósito de probar las relaciones establecidas, en el marco de un diseño de investigación transeccional o transversal y tipos: descriptivo y correlacionalcausal, entendiendo que el diseño no es sino un "plan o estrategia", establecido para recabar información requerida en una investigación; en un solo momento, describiendo 
el comportamiento de las variables y las relaciones de las mismas (Hernández, Fernández y Baptista, 2014).

Modelo 1

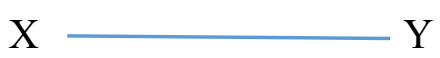

Modelo 2

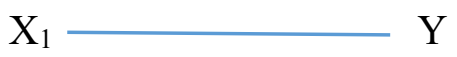

Modelo 3

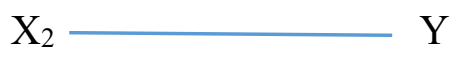

Modelo 4

$\mathrm{X}_{3} \longrightarrow \mathrm{Y}$

Modelo 5

$\mathrm{X}_{4} \longrightarrow \mathrm{Y}$

Donde:

$\mathrm{X}$ : Liderazgo del director; $\mathrm{X}_{1}$ : Liderazgo educativo; $\mathrm{X}_{2}$ : Liderazgo situacional; $\mathrm{X}_{3}$ : Liderazgo innovador; $\mathrm{X}_{4}$ : Liderazgo estratégico; Y: Clima escolar; $\mathrm{Y}_{1}$ : Comunicación institucional; $\mathrm{Y}_{2}$ : Confianza institucional; $\mathrm{Y}_{3}$ : Motivación institucional; $\mathrm{Y}_{4}$ : Participación institucional

\section{Población y criterios de selección}

La investigación se realizó en la Asociación Educativa Sur Oriental del Perú, comprende tres departamentos: Madre de Dios, Apurímac y Cusco, en ocho colegios: dos del primer departamento, uno del segundo y cinco del tercero. Participaron los docentes de los tres niveles: inicial, primerio y secundario, cuya suma total es 175 , determinada por conveniencia y por la estructura ya establecida orgánica, administrativa y legalmente. Sin embargo, solamente se consideraron 172 encuestas y tres fueron eliminadas, debido a fueron mal llenadas, razón por la cual solamente en los resultados aparece el número 172.

\section{Técnicas de recolección de datos}

En la investigación se abordó dos variables: liderazgo del director y clima escolar. Para recabar los datos requeridos, se construyeron dos instrumentos, sobre la base de dos principios: los teóricos y los de validación. Uno fue diseñado para medir el liderazgo del director y otro para evaluar el clima escolar. Ambos instrumentos tienen una estructura 
común: “I. INFORMACIÓN DEMOGRÁFICA, la cual tiene las variables: género, edad, cargo desempeñado, área curricular, nivel donde labora y el nombre de la institución.

Se utilizó la encuesta en la condición de técnica, con sus respectivos instrumentos. Para el instrumento del liderazgo del director, la segunda parte es denominada: "II. INFORMACIÓN SOBRE EL LIDERAZGO DEL DIRECTOR”; la variable: liderazgo del director presenta cinco dimensiones: liderazgo educativo, liderazgo situacional, liderazgo innovador, liderazgo técnico y liderazgo estratégico. Se construyó la escala al tipo Likert, con la nomenclatura lingüística:

\begin{tabular}{|c|c|c|c|}
\hline 1 & 2 & 3 & 4 \\
\hline $\begin{array}{c}\text { Completamente } \\
\text { en desacuerdo }\end{array}$ & En desacuerdo & De acuerdo & Completamente de acuerdo \\
\hline
\end{tabular}

El instrumento tiene 28 ítems, organizados en las cinco dimensiones: liderazgo educativo (10 ítems: 1, 2, 3, 4, 5, 6, 7, 8, 9 y 10), liderazgo situacional (6 ítems: 11, 12, 13, 14, 15 y 16), liderazgo innovador (4 ítems: 17, 18, 19 y 20), liderazgo técnico (4 ítems: 21, 22, 23 y 24) y liderazgo estratégico (4: ítems: 25, 26, 27 y 28). La segunda parte del instrumento para la evaluación del clima institucional ha sido denominada: "II. INFORMACIÓN SOBRE EL CLIMA INSTITUCIONAL”. El clima institucional, la variable, presenta cuatro dimensiones: comunicación institucional, confianza institucional, motivación institucional y participación institucional; las dimensiones fueron evaluadas mediante la escala tipo Likert, con la estructura lingüística:

\begin{tabular}{|c|l|l|l|}
\hline 1 & 2 & 3 & 4 \\
\hline $\begin{array}{l}\text { Completamente } \\
\text { en desacuerdo }\end{array}$ & En desacuerdo & De acuerdo & Completamente de acuerdo \\
\hline
\end{tabular}

Este instrumento presenta 34 ítems, organizados en las cuatro dimensiones: comunicación institucional (12 ítems: 1, 2, 3, 4, 5, 6, 7, 8, 9, 10, 11 y 12), confianza institucional (7 ítems: 13, 14, 15, 16, 17, 18 y 19), motivación institucional (6 ítems: 20, $21,22,23,24$ y 25) y participación institucional (9 ítems: 26, 27, 28, 29, 30, 31, 32, 33 y 34).

\section{Plan de análisis de datos}

Con el propósito de generar los datos y las condiciones, necesarios para los respectivos análisis, se han considerados los protocolos institucionales y las consignas investigativas establecidas por la universidad y por las normas internacionales. Los datos 
requeridos han sido generados mediante el uso de la estadística descriptiva, gracias a la cual se han generado y analizado los datos que corresponden a la información demográfica: género, edad, cargo desempeñado, área curricular, nivel donde labora y nombre de la institución. Los datos y la información requerida son presentados mediante tablas, cuyos usos son convencionales y estandarizados según el APA.

Por otro lado, se ha utilizados la estadística inferencial, con el propósito de analizar y probar las diversas hipótesis planificadas.

\section{Validez y confiabilidad}

Para lograr los efectos de la validez y la confiabilidad de los instrumentos, se ha recurrido también a protocolos ya establecidos en el universo de las investigaciones. Los instrumentos (para la medición del liderazgo del director y para la evaluación del clima institucional) fueron elaborados siguiendo los procedimientos: a) elaboración de los instrumentos sobre la base de las revisiones teóricas, cuyas variables tengan coherencia, pertinencia y consistencia con las dimensiones y los ítems; b) revisión preliminar de los instrumentos por el investigador y el profesor guía; c) elección de los profesionales para la emisión de juicio de expertos; d) solicitud del juicio de expertos y e) la prueba piloto.

Tabla 1

Prueba de confiabilidad

Estadísticas de fiabilidad

Alfa de Cronbach $\quad \mathrm{N}$ de elementos

$\begin{array}{r}\hline, 977 \\ \hline\end{array}$

El resultado del análisis de confiabilidad de Alfa de Cronbach arrojó un resultado de $97.7 \%$ de confiabilidad; en efecto, este un resultado altamente confiable. 


\section{Resultados}

Prueba de correlación: liderazgo educativo del director y el clima institucional

Tabla 2.

Prueba de correlación Rho de Spearman entre el liderazgo educativo del director y el clima institucional

\begin{tabular}{lccc}
\hline & \multicolumn{3}{c}{ Clima institucional } \\
& $\begin{array}{c}\text { Rho } \\
\text { Spearman }\end{array}$ & $\begin{array}{c}\text { Sig. } \\
\text { (bilateral) }\end{array}$ & N \\
\hline $\begin{array}{l}\text { Liderazgo } \\
\text { educativo }\end{array}$ & $\mathbf{5 6 7 7 ^ { * * }}$ & $\mathbf{. 0 0 0}$ & $\mathbf{1 5 6}$ \\
\hline & & & \\
\hline$* *$. La correlación es significativa en el nivel 0,01 (2 colas).
\end{tabular}

Después del análisis estadístico de correlación de Rho Spearman, se obtuvo el resultado de 0,567 y un p valor igual a 0.000 ( $\mathrm{p}$ valor $<0,05$ ); por lo tanto, se rechaza la hipótesis nula y se acepta la hipótesis de investigación; el liderazgo educativo del director se relaciona, en forma significativa, con el clima institucional. Además, se observa que la relación entre las variables estudiadas es directa; vale decir que, a mejor liderazgo educativo del director, mejor será el clima institucional.

Prueba de correlación: liderazgo situacional del director y el clima institucional

Tabla 3.

Prueba de correlación Rho de Spearman entre el liderazgo situacional del director y el clima institucional

\begin{tabular}{lccc}
\hline & \multicolumn{3}{c}{ Clima institucional } \\
& $\begin{array}{c}\text { Rho } \\
\text { Spearman }\end{array}$ & $\begin{array}{c}\text { Sig. } \\
\text { (bilateral) }\end{array}$ & $\mathrm{N}$ \\
\hline $\begin{array}{l}\text { Liderazgo } \\
\text { situacional }\end{array}$ & $\mathbf{5 2 3}^{* *}$ & $\mathbf{. 0 0 0}$ & $\mathbf{1 5 6}$ \\
\hline **. La correlación es significativa en el nivel 0,01 (2 colas).
\end{tabular}

Hecho el análisis estadístico de correlación de Rho Spearman se obtuvo el resultado de 0,523 y un $p$ valor igual a 0.000 ( $p$ valor $<0,05$ ); por lo tanto, se rechaza la hipótesis nula y se acepta la hipótesis de investigación; es decir, el liderazgo situacional del director 
se relaciona, en forma significativa, con el clima institucional; además se observa que la relación entre las variables estudiadas es directa; en otras palabras, a mejor liderazgo situacional del director, mejor será el clima institucional.

\section{Prueba de correlación: liderazgo innovador del director y el clima institucional}

Tabla 4.

Prueba de correlación Rho de Spearman entre el liderazgo innovador del director y el clima institucional

\begin{tabular}{lccc}
\hline & \multicolumn{3}{c}{ Clima institucional } \\
& $\begin{array}{c}\text { Rho } \\
\text { Spearman }\end{array}$ & $\begin{array}{c}\text { Sig. } \\
\text { (bilateral) }\end{array}$ & $\mathrm{N}$ \\
\hline $\begin{array}{l}\text { Liderazgo } \\
\text { innovador }\end{array}$ &, $527^{* *}$ & .000 & 156 \\
\hline **. La correlación es significativa en el nivel 0,01 (2 colas).
\end{tabular}

Después del análisis estadístico de correlación de Rho Spearman, se obtuvo el resultado de 0,527 y un $\mathrm{p}$ valor igual a 0.000 ( $\mathrm{p}$ valor $<0,05$ ); por lo tanto, se rechaza la hipótesis nula y se acepta la hipótesis de investigación; vale decir, el liderazgo innovador del director se relaciona, en forma significativa, con el clima institucional. Además, se observa que la relación entre las variables estudiadas es directa; es decir, a mejor liderazgo innovador del director, mejor será el clima institucional.

\section{Prueba de correlación: liderazgo técnico del director y el clima institucional}

Tabla 5.

Prueba de correlación Rho de Spearman entre el liderazgo técnico del director y el clima institucional

\section{Clima institucional}

\begin{tabular}{lrrrr} 
& $\begin{array}{c}\text { Rho } \\
\text { Spearman }\end{array}$ & $\begin{array}{c}\text { Sig. } \\
\text { (bilateral) }\end{array}$ & N & \\
\hline $\begin{array}{l}\text { Liderazgo } \\
\text { técnico }\end{array}$ &, $484^{* *}$ & .000 & 156 \\
\hline
\end{tabular}

\footnotetext{
**. La correlación es significativa en el nivel 0,01 (2 colas).
}

Luego del análisis estadístico de correlación de Rho Spearman, se obtuvo el resultado de 0,408 y un $p$ valor igual a 0.000 ( $\mathrm{p}$ valor $<0,05$ ); por lo tanto, se rechaza la hipótesis 
nula y se acepta la hipótesis de investigación; en otras palabras, el liderazgo técnico del director se relaciona, en forma significativa, con el clima institucional. También se observa que la relación entre las variables estudiadas es directa; o sea, a mejor liderazgo técnico del director, mejor será el clima institucional.

\section{Prueba de correlación: liderazgo estratégico del director y el clima institucional}

Tabla 6.

Prueba de correlación Rho de Spearman entre el liderazgo estratégico del director y el clima institucional

\begin{tabular}{|c|c|c|c|}
\hline & \multicolumn{3}{|c|}{ Clima institucional } \\
\hline & $\begin{array}{c}\text { Rho } \\
\text { Spearman }\end{array}$ & $\begin{array}{c}\text { Sig. } \\
\text { (bilateral) }\end{array}$ & $\mathrm{N}$ \\
\hline $\begin{array}{l}\text { Liderazgo } \\
\text { estratégico }\end{array}$ &, $567^{* *}$ & .000 & 156 \\
\hline
\end{tabular}

**. La correlación es significativa en el nivel 0,01 (2 colas).

Hecho el análisis estadístico de correlación de Rho Spearman, se obtuvo el resultado de 0,567 y un $\mathrm{p}$ valor igual a 0.000 ( $\mathrm{p}$ valor $<0,05$ ); por lo tanto, se rechaza la hipótesis nula y se acepta la hipótesis de investigación; es decir, el liderazgo estratégico del director se relaciona, en forma significativa, con el clima institucional. Además, se observa que la relación entre las variables estudiadas es directa; dicho de otro modo, a mejor liderazgo estratégico del director, mejor será el clima institucional.

Prueba de correlación: liderazgo del director y el clima institucional

Tabla 7.

Prueba de correlación Rho de Spearman entre el liderazgo del director y el clima institucional

\begin{tabular}{|c|c|c|c|}
\hline & \multicolumn{3}{|c|}{ Clima institucional } \\
\hline & $\begin{array}{c}\text { Rho } \\
\text { Spearman }\end{array}$ & $\begin{array}{c}\text { Sig. } \\
\text { (bilateral) }\end{array}$ & $\mathrm{N}$ \\
\hline $\begin{array}{l}\text { Liderazgo del } \\
\text { director }\end{array}$ &, $573^{* *}$ & .000 & 156 \\
\hline
\end{tabular}


El análisis estadístico de correlación de Rho Spearman, dejó el resultado de 0, 582 y un p valor igual a $0.000(<0,05)$; por lo tanto, se rechaza la hipótesis nula y se acepta la hipótesis de investigación; es decir, el liderazgo del director se relaciona, en forma significativa, con el clima institucional. Además, se observa que la relación entre las variables estudiadas es directa; expresado de otro modo, vale decir que, a mejor liderazgo del director, mejor será el clima institucional.

\section{Discusión}

Cabezudo y García (2016) estudiaron el liderazgo y el clima organizacional, en la comunidad shipiba, con el objetivo de estudiar la relación de estas variables, en 42 profesores del nivel primario, con un enfoque cuantitativo y los parámetros de una investigación básica, descriptiva, correlacional. En sus resultados, la "r" de Spearman es 0,630 , significa que existe correlación positiva y fuerte. Además, "se concluye que existe una relación directa y significativa media entre el liderazgo y clima Organizacional”. Al comparar, a diferencia de la anterior, el presente estudio se realizó en los tres niveles: inicial, primario y secundario, según el sistema educativo peruano; también se realizó en una comunidad de habla hispana, a diferencia de la anterior, cuya comunidad es shipiba; además realizaron la investigación en 42 docentes; en cambio, la investigación presente tuvo la participación de 172 docentes. Significa que el universo de esta investigación es mucho mayor y más amplio, cuyo estadístico de correlación de Rho Spearman presenta el resultado de 0,573 , lo que significa que, en comparación con la antecesora, la correlación es menor a 0,630. En cambio, la metodología es totalmente semejante en las dos investigaciones; es decir, son básicas, descriptivas y correlacionales. Oré y Riversos (2017) realizaron su investigación en el departamento de Ica (Perú), abordando el liderazgo directivo y el clima organizacional, con el objetivo de "relacionar el liderazgo directivo y el clima organizacional en las instituciones educativas del nivel inicial $\mathrm{N}^{\circ} 151$ y 157 Palpa-Ica”, recurriendo a un estudio cuantitativo, trabajando la correlación de las variables, realizando además descripciones de los datos, cuyos datos fueron tomados en un solo momento, ejecutando la transversalidad. Los resultados revelan que el liderazgo directivo, en las instituciones educativas, presenta tres realidades: "solamente existe" en "un 27,49\%"; "a veces existe en un 40,57\%"; y "no existe" "en un 31,81\%". Estos resultados prueban la hipótesis; sin embargo, la conclusión general confirma que no existe relación entre las variables referidas 
En esta discusión, se refiere que la hipótesis general de la presente investigación tiene mucha semejanza con el estudio que antecede; por ejemplo, esta hipótesis es: "el liderazgo del director se relaciona, en forma significativa, con el clima institucional, en la Asociación Educativa Sur Oriental del Perú", con la diferencia de que se ha trabajado en varias instituciones educativas.

Además, la metodología es semejante, con la diferencia en los resultados. Se determinó la relación entre las variables: liderazgo del director y clima institucional, cuyo análisis estadístico de correlación de Rho Spearman presenta el resultado de 0,573 y un $p$ valor igual a $0.000(<0,05)$. En consecuencia, se rechazó la hipótesis nula y se aceptó la hipótesis de investigación. En definitiva, el liderazgo del director se relaciona, en forma significativa, con el clima institucional. La relación entre las variables estudiadas es directa; esto significa que, a mejor liderazgo del director, mejor será el clima institucional. Por otro lado, Contreras y Jiménez, (2016) realizaron su estudio con el objetivo de analizar el liderazgo directivo y el clima organizacional, respondiendo a motivos de intervención, cuyo estudio fue descriptivo, además dichas variables fueron valuadas mediante el uso de dos instrumentos, uno para cada variable, "el Test de Adjetivos de Pitcher (PAT) (Pitcher, 1997) y la Escala del Clima Organizacional (ECO) (Fernándes, 2008), respectivamente". El estudio se realizó “con los directivos, los docentes y los estudiantes de los grados noveno, décimo y undécimo del Colegio". El estudio, por otro lado, también revela ciertas dificultades encontradas en el liderazgo y algunos problemas en clima organizacional, según las luces de las teorías y las prácticas.

También Niebla y Ibarra (2017) investigaron el liderazgo directivo femenino y el clima organizacional, dicho estudio se realizó, Escuela Preparatoria Central Nocturna de la zona centro de Sinaloa, en una escuela de educación media superior, con el propósito de "explorar y describir el liderazgo directivo femenino y el clima organizacional", mediante la medición de dos instrumentos: uno para la directora y otro para los docentes, evaluando el liderazgo de la directora; para evaluar el clima organizacional, se utilizó una encuesta; el estudio fue exploratorio-descriptivo, con un diseño transversal, con la participación de la directora, por un lado; por otro, con la participación de 36 profesores. Según la percepción de los docentes, la directora tiene la tendencia a dirigir; además refirieron que el clima no es óptimo.

En comparación con la presente investigación, el estudio se dio con varios directores y con número mucho mayor de docentes: 172, quienes forman parte de los tres 
niveles: inicial, primario y secundario. En esta parte, además, es conveniente declarar que la investigación se realizó en instituciones educativas particulares, donde se encuentra la presencia de la promotora: Iglesia Adventista del Séptimo Día, cuyo universo pedagógico y administrativo es clarificado por la filosofía de la educación cristiana y por la práctica e interiorización de los valores bíblico-cristianos, los cuales dejan una atmósfera y un clima totalmente diferentes a los encontrados en otras instituciones educativas sin la misma filosofía. Por otro lado, la investigación presente no fue exploratoria, ni descriptiva, sí transversal y correlacional.

\section{Conclusiones}

El liderazgo directivo se relaciona, en forma significativa, con el clima institucional, de acuerdo con el análisis estadístico de correlación de Rho Spearman, cuyo resultado es 0,573 y un p valor igual a $0.000(<0,05)$, suscribiendo que la relación, además, es directa; vale decir que, a mejor liderazgo del director, mejor será el clima institucional.

El liderazgo educativo del director se relaciona, de manera significativa, con el clima institucional, según el análisis estadístico de correlación de Rho Spearman, reflejada en resultado de 0,567 y un p valor igual a $0.000(<0,05)$. También vale decir que, a mejor liderazgo educativo del director, mejor será el clima institucional.

Por otro lado, el liderazgo situacional del director se relaciona, significativamente, con el clima institucional, la cual además de significativa es directa, a la luz del análisis estadístico de correlación de Rho Spearman, cuyo resultado es 0,523 y un p valor igual a $0.000(<0,05)$. Significa también que, a mejor liderazgo situacional del director, mejor será el clima institucional.

El liderazgo innovador del director se relaciona, en forma significativa, con el clima institucional; en este sentido, se concluye que existe relación significativa y directa entre las variables, en armonía con el análisis estadístico de correlación de Rho Spearman que dejó el resultado de 0,527 y un p valor igual a $0.000(<0,05)$. Por otro lado, se puede decir: a mejor liderazgo innovador del director, mejor será el clima institucional.

El liderazgo técnico del director se relaciona, en forma significativa, con el clima institucional; es decir, se concluye que la relación entre las variables es significativa y también directa, según el análisis estadístico de correlación de Rho Spearman, cuyo 
resultado es 0,408 y un p valor igual a $0.000(<0,05)$; también se afirma que, a mejor liderazgo técnico del director, mejor será el clima institucional.

El liderazgo estratégico del director se relaciona, significativamente, con el clima institucional, cuya relación es significativa y directa, de acuerdo con el análisis estadístico de correlación de Rho Spearman, expresado en el resultado de 0,567 y un p valor igual a $0.000(<0,05)$; además, a mejor liderazgo estratégico del director, mejor será el clima institucional.

\section{Referencias}

Aparicio, C., Sepúlveda, F., Valverde, X., Cárdenas, V., Contreras, G. y Valenzuela, M. (2020). Liderazgo directivo y cambio educativo: Análisis de una experiencia de colaboración Universidad-Escuela. Páginas de Educación, 13(1), 19-41.

https://doi.org/10.22235/pe.v13i1.1915

Arca, D. C. L. (2020). Plan de marketing interno para mejorar el clima organizacional de los trabajadores del departamento de cobranzas de la Empresa Servicios Cobranzas e Inversiones S.A.C, Chiclayo [Tesis de maestría, Universidad Señor de Sipán]. https://repositorio.unprg.edu.pe/bitstream/handle/20.500.12893/7006/BC322 CORDOVA PINTADO.pdf? sequence $=1 \&$ isAllowed $=\mathrm{y}$

Cabezudo, M. M. y García, R. (2016). Liderazgo y Clima Organizacional de los docentes de las Instituciones Educativas Primarias Públicas de las Comunidades Shipibas de Yarinacocha-2015 . [Tesis, Universidad Nacional Intercultural de la Amazonia]. http://repositorio.unia.edu.pe/bitstream/unia/61/1/Informe de Tesis de Melissa Cabezudo - 2016 corregido.pdf

Calderón, F. A. (2017). Relación entre liderazgo y clima institucional en docentes del Instituto Superior Pedagógico Público “Cachicadán” Santiago de Chuco, La Libertad [Tesis de maestría, Universidad Nacional Pedro Ruiz Gallo.]. https://repositorio.unprg.edu.pe/bitstream/handle/20.500.12893/8826/Calderon_Ca $\underline{\text { rlos_Francisco_Artemio.pdf? sequence }=1 \& \text { isAllowed }=\mathrm{y}}$

Chicoma, M. M. (2018). Estrategias metodológicas eficaces para el proceso de enseñanza-aprendizaje en el área de matemática. [Trabajo académico para optar el título de segunda especialidad, Pontificia Universidad Católica del Perú]. https://tesis.pucp.edu.pe/repositorio/handle/20.500.12404/11404 
Contreras, D. M. y Jiménez, L. F. (2016). Liderazgo y clima organizacional en un colegio de Cundinamarca. Lineamientos básicos para su intervención. [Trabajo de grado, Universidad del Rosario.].

https://repository.urosario.edu.co/bitstream/handle/10336/11983/ContrerasRiosDoris-2016.pdf? sequence $=9 \&$ isAllowed $=\mathrm{y}$

Córdova, E., Rojas, I. y Marín, S. (2021). El liderazgo directivo de las instituciones educativas: una revisión bibliográfica. Revista Conrado, 17(80), 231-236. http://scielo.sld.cu/pdf/rc/v17n80/1990-8644-rc-17-80-231.pdf

Còrdova, H. E. (2015). Estrategia de liderazgo sustentado en la teoría de Frederick Herzberg, Thomas y Velthouse, Litwin y Stringer para mejorar el clima laboral de la institución educativa $N^{\circ} 15135$ del caserío las Lomas, Pacaipampa, Ayabaca, año 2014 [Tesis de maestría, Universidad Nacional Pedro Ruiz Gallo]. https://repositorio.unprg.edu.pe/bitstream/handle/20.500.12893/7006/BC-322 $\underline{\text { CORDOVA PINTADO.pdf? sequence }=1 \& \text { isAllowed }=\mathrm{y}}$

Cortez, A. D. (2018). Indicadores de comunicación asertiva del docente y la generación del clima escolar en el aula en situaciones de aprendizaje. [Tesis de maestría, Universidad Andina Simón Bolívar].

https://repositorio.uasb.edu.ec/bitstream/10644/6221/1/T2652-MIE-CortezIndicadores.pdf

Cuenca, R. y Cáceda, J. (2017). Ideales normativos, normas y praxis: patologías sociales sobre los directivos escolares en el Perú. REICE. Revista Iberoamericana Sobre Calidad, Eficacia y Cambio En Educación, 15(2), 5-29. https://doi.org/10.15366/reice2017.15.2.001

Delhom, I., Satorres, E. y Meléndez, J. C. (2019). ¿Están los rasgos de personalidad asociados al bienestar psicológico? Escritos de Psicología / Psychological Writings, 12(1), 1-8. https://doi.org/10.5231/psy.writ.2019.0107

González, R. (2017). Detección del liderazgo como factor detonante de un buen clima organizacional en Educación Media Superior. https://repositorio.itesm.mx/ortec/handle/11285/622499

Gutierrez, Y. E. (2020). Liderazgo directivo y su relación con el desempeño docente. 
Ciencia y Educación, 1(4), 27-36.

http://www.cienciayeducacion.com/index.php/journal/article/view/22/37

Hernández Sampieri, R., Fernández Collado, C., \& Baptista Lucio, M. del P. (2014). Metodología de la Investigación (Sexta edic). McGraw-Hill Interamericana Editores, S.A. de C.V.

Herrera, S. R. y Tobón, S. (2017). El director escolar desde el enfoque socioformativo. Estudio documental mediante la cartografía conceptual. Revista de Pedagogía, 38(102), 164-194. http://www.redalyc.org/pdf/659/65952814009.pdf

Horn, A. y Murillo, F. (2016). Incidencia de la dirección escolar sobre el compromiso de los docentes: Un estudio multinivel. Psicoperspectivas. Individuo y Sociedad, 15(2), 64-77. https://doi.org/10.5027PSICOPERSPECTIVASVOL15-ISSUE2FULLTEXT-746

Lescano, L. (2017). Cómo fortalecer el clima y la cultura de servicio a través del liderazgo de servicio del mando intermedio: caso de estudio en una compañía multinacional. Revista Empresa y Humanismo, XX(1), 65-96. https://doi.org/10.15581/015.XX.1.65-96

Madero, S. M. y Rodríguez, D. R. (2018). Relación entre las teorías X y Y de McGregor, las formas de retribuir y la satisfacción de las personas en su trabajo. CienciaUAT, 13(1), 95-107. https://doi.org/doi.org/10.29059/cienciauat.v13i1.1014

Marlene, C. y Rujel, E. M. (2020). Factores que permiten identificar el uso de la teoría “ $X$ "o " $Y$ ” de Mc Gregor en la empresa Oechsle que opera en el centro comercial real plaza centro cívico en el periodo 2020 [Tesis, Universidad Tecnológica del Perú]. https://repositorio.utp.edu.pe/handle/20.500.12867/3922

Maya, E., Aldana, J. J. y Isea, J. (2019). Liderazgo Directivo y Educación de Calidad. CIENCIAMATRIA Revista Interdisciplinaria de Humanidades, Educación, Ciencia y Tecnología, $V(9), 114-129$.

https://repositorio.uasb.edu.ec/bitstream/10644/6221/1/T2652-MIE-CortezIndicadores.pdf 
Medina, A. V. (2018). Diseño de una metodología basada en la teoría de sistemas, para superar las deficiencias en la gestión administrativa del Instituto Superior Pedagógico “Monseñor Francisco Gonzáles Burga” de Ferreñafe [Tesis de maestría, Universidad Nacional Pedro Ruiz Gallo]. https://repositorio.unprg.edu.pe/bitstream/handle/20.500.12893/7273/BCMEDINA CHAVEZ.pdf?sequence $=1 \&$ isAllowed $=\mathrm{y}$

Mejía, C. (2015). Estilos de liderazgo y su impacto en la participación de los docentes de la institución educativa coronel José Joaquín Inclán de Piura. [Tesis de Maestría, Universidad de Piura]. https://pirhua.udep.edu.pe/bitstream/handle/11042/3185/MAE_EDUC 136.pdf?se quence $=1 \&$ isAllowed $=\mathrm{y}$

Naira, E. (2018). Establecer un sistema de gestión de la convivencia escolar que involucre la participación de estudiantes, docentes y padres de familia del $V$ ciclo de la I.E 15110 “José Gabriel Condorcanqui” del distrito veintiséis de octubrePiura. [Tesis, Pontificia Universidad Católica del Perú]. https://tesis.pucp.edu.pe/repositorio/handle/20.500.12404/10520

Navarro, J. D. (2018). Programa de estrategias de liderazgo basado en la teoría de LIKERT para mejorar el clima institucional en la Institución Educativa $N^{\circ} 10834$ del distrito de José Leonardo Ortiz - Región Lambayeque. [Tesis de grado de maestra, Universidad Nacional Pedro Ruiz Gallo]. https://repositorio.unprg.edu.pe/handle/20.500.12893/8654

Niebla, S. Y. y Ibarra, E. (2017). Liderazgo directivo femenino y el clima organizacional en una escuela de educación media superior. Praxis Investigativa ReDIE, 9(17), 42-52. http://repositorio.unsm.edu.pe/handle/11458/2816

Ordoñez, C. G., Castillo, D. C., Ordoñez, A. L. del R. y Orbe, M. P. (2020). Liderazgo directivo y desempeño docente: Abordaje desde el ámbito legal ecuatoriano. Journal of Business and Entrepreneurial Studies., 4(1), 1-12. http://www.redalyc.org/articulo.oa?id=573661266016

Oré, A. y Riversos, K. (2017). Liderazgo directivo y el clima organizacional en las instituciones educativas del nivel inicial $N^{\circ} 151$ y 157 - Palpa - Ica [(Tesis para 
optar el título de segunda especialidad profesional). Universidad Nacional de Huancavelica, Facultad de Educación. Huancavelica, Perú.]. https://doi.org/Doi $\underline{10.1590 / \mathrm{S} 0100-40422005000200003}$

Peralta, E. (2016). Teoría general de los sistemas aplicada a modelos de gestión. Aglala, 7(1), 122-146. https://doi.org/10.22519/22157360.901

Puscán, G. (2017). Programa socioemocional para mejorar el clima escolar en estudiantes del segundo grado de educación secundaria en la IE "Ignacia Velásquez”-Moyobamba. [Tesis de maestría, Universidad Nacional de San Martin]. http://repositorio.unsm.edu.pe/handle/11458/2816

Quezada, S. (2018). El liderazgo estratégico del director y el éxito institucional del programa de tecnología educativa de la Universidad Nacional de Trujillo-2016 [Tesis doctoral, Universidad Privada Antenor Orrego]. http://repositorio.upao.edu.pe/bitstream/upaorep/3784/1/RE_DOCT_EDU_SONIA .QUEZADA LIDERAZGO.ESTRATEGICO DATOS.PDF

Rodríguez, C. L., Torres, C. y Acosta, A. M. (2020). Liderazgo directivo para la justicia social en contextos vulnerables: estudio de caso de directores escolares mexicanos. Perspectiva Educacional, 59(2), 4-26. https://doi.org/10.4151/07189729-vol.59iss.2-art.1025

Rossi, R. M. y Rossi, R. G. (2021). Liderazgo directivo, modelo de medida del constructo para aplicación en Educación Básica regular. Revista Andina de Educación, 4(1), 11-22. https://doi.org/10.32719/26312816.2021.4.1.2

Ruiz, B. L. (2016). Promoción de un clima escolar favorable para la mejora de los aprendizajes Plan de acción. Pontificia Universidad Católica del Perú, Facultad de Educación.

Salvador, E. M.y Sánchez, J. A. (2018). Liderazgo de los directivos y compromiso organizacional docente. Journal of High Andean Research, 20(1), 115-124. http://www.scielo.org.pe/pdf/ria/v20n1/a11v20n1.pdf

Simbron, S. F. y Sanabria, F. M. (2020). Liderazgo directivo, clima organizacional y satisfacción laboral del docente. Cienciamatria, VI(1), 59-83. 
https://doi.org/10.35381/cm.v6i10.295

Valdéz, Á. A., Tánori, J., Sotelo, T. I. y Ochoa, J. A. (2018). Práticas docentes, clima social, segurança escolar e violência entre estudantes. Magis, Revista Internacional de Investigación En Educación, 10(21), 109-120.

https://doi.org/10.11144/Javeriana.m10-21.pdes

Valdivia, R. R. (2018). Evaluación de la gestión de la dirección asociada al liderazgo organizacional en docentes de las instituciones educativas saludables de la Red Arequipa - Caylloma, 2016. [Tesis de maestro, Universidad Nacional Pedro Ruiz Gallo]. https://repositorio.unprg.edu.pe/handle/20.500.12893/7253

Vargas, A. C. y Vásquez, A. (2018). Propuesta de liderazgo según teoría X-Y de McGregor para desarrollar la motivación en docentes. Revista Hacedor, 2(2), 1832. http://revistas.uss.edu.pe/index.php/HACEDOR/article/view/981/841

Vega, M. A. (2017). Convvencia escolar: dimensiones, programas y evaluación. [Tesis, doctoral, Universidad Pública de Navarra]. https://academicae.unavarra.es/xmlui/bitstream/handle/2454/28899/Tesis doctoral Ma Asunción $\underline{\text { Vega Osés.pdf? } \text { sequence }=1 \& \text { isAllowed }=\mathrm{y}}$ 